\title{
Closed-Loop Perching and Spatial Guidance Laws for Bio-Inspired Articulated Wing MAV
}

\author{
Aditya A. Paranjape, Joseph Kim†, and Soon-Jo Chung $\ddagger$ \\ Department of Aerospace Engineering, \\ University of Illinois at Urbana-Champaign, Urbana, IL 61801.
}

This paper presents the underlying theoretical developments and successful experimental demonstrations of perching of an aerial robot. The open-loop lateral-directional dynamics of the robot are inherently unstable because it lacks a vertical tail for agility, similar to birds. A unique feature of this robot is that it uses wing articulation for controlling the flight path angle as well as the heading. New guidance algorithms with guaranteed stability are obtained by rewriting the flight dynamic equations in the spatial domain rather than as functions of time, after which dynamic inversion is employed. It is shown that nonlinear dynamic inversion naturally leads to proportional-integral-derivative (PID) controllers, thereby providing an exact method for tuning the gains. The effectiveness of the proposed bio-inspired robot design and its novel closed-loop perching controller has been successfully demonstrated with perched landings on a human hand.

\section{Introduction}

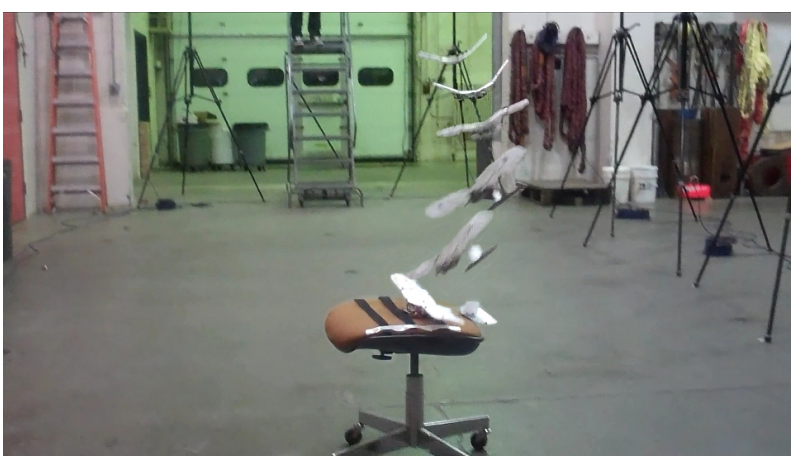

(a) Perched landing on a chair

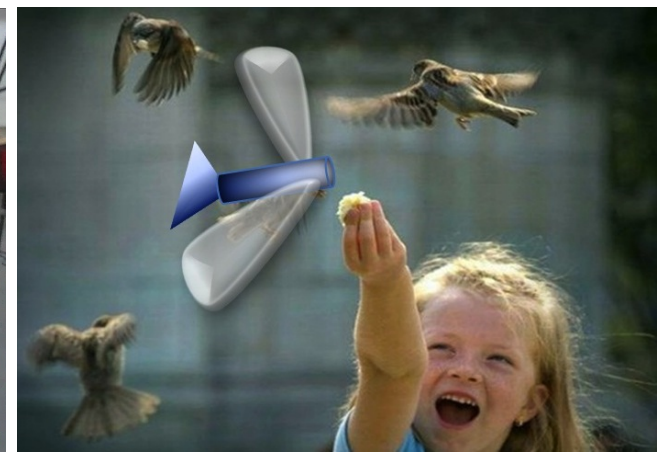

(b) Operations in close vicinity of humans that might need perching

Figure 1. The first picture shows a perched landing on a chair performed by the aerial robot designed by the authors. The second picture is a schematic showing an aerial robot performing operating in close vicinity to humans. The latter is a benchmark application for perching. (Source for the second picture: 500px.com, author: Didier Cauvain, available at http://500px.com/photo/564564)

There is a growing interest in developing bio-inspired robotic aircraft. While most prior works use insects as their primary models, ${ }^{1-5}$ bird-sized micro aerial vehicles (MAVs) ${ }^{6,7}$ offer several unique features including a capability to switch between flapping flight and gliding flight. The driving philosophy behind the present

*Post-Doctoral Research Associate. Email: paranja2@illinois.edu; Member, AIAA.

$\dagger$ Undergraduate Student. Email: kim323@illinois.edu; Student Member, AIAA.

$\ddagger$ Assistant Professor. Also with the Coordinated Science Laboratory. Email: sjchung@illinois.edu; Senior Member, AIAA. 


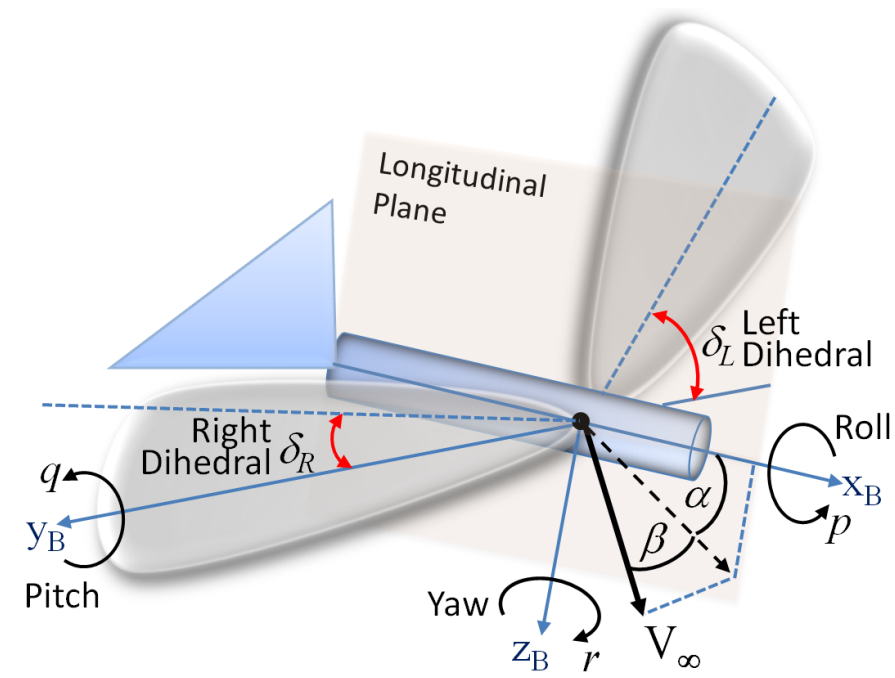

Figure 2. Common notations for the body axes of the flying vehicle (see Table 1). The up-and-down angle of the wing $\left(\delta_{R}, \delta_{L}\right)$, called the dihedral angle, measures the elevation of the wing with respect to the $y$ axis.

paper is that the maneuverability and control efficiency of avian flight can be replicated by applying their actuation and control principles to advanced MAVs designed on the size scale of small birds.

The flight regime of birds is also interesting because their lift-to-drag (L/D) ratios are suitable for occasional gliding. Indeed, gliding is used for soaring, descending, and landing. The wings of ornithopters are inherently capable of being reoriented, and this capability can be used for controlling and maneuvering the aircraft in a gliding phase, thereby eliminating the need for additional traditional actuators. In this paper, we describe an articulated-wing-based concept for an agile robotic aircraft inspired by birds. This concept is meant to be implemented on flapping wing aircraft.

Of all maneuvers executed by flapping wing aircraft in a gliding phase, a perched landing is arguably the most challenging. The primary reason is twofold: (1) its duration is very short, on the same order as the aircraft dynamics, and (2) a high level of position accuracy is required for a successful perched landing. The aerial robot concept proposed in the paper lacks a vertical tail for improved agility, similar to birds, which renders it dynamically unstable and exacerbates both challenges listed above. Consequently, we choose a perching maneuver to demonstrate the capabilities of our articulated-winged aircraft concept and novel guidance algorithms and control design. In particular, the ability to perform perched landings on a human hand endows our robot with the ability to operate around humans (see Fig. 1).

In the interests of ensuring a coherent presentation, we now introduce the standard notation used in flight dynamics. Thereafter, we will review the literature on perching and highlight the contributions of our work.

\section{A. Nomenclature and Preliminaries}

Aircraft motion is traditionally split into two categories: motion in its nominal plane of symmetry (marked as the $x_{B}-z_{B}$ plane in Fig. 2), called the longitudinal motion, and motion outside the plane of symmetry, called the lateral-directional motion. Figure 2 also shows some important flight dynamic parameters. Note that rotations about $x_{B}, y_{B}$ and $z_{B}$ axes are called roll, pitch, and yaw, respectively. The list of the longitudinal and lateral-directional variables is given in Table 1.

\section{B. Motivation from Nature}

Birds and some species of bats spend a considerable amount of their flight time in either low frequency flapping or gliding flight, particularly while soaring, descending, or executing a perched landing. Perching is routinely used by birds to land on objects such as tree branches, power wires, building ledges, etc. The design of a typical perching maneuver is inspired by that of birds. Figure 3 shows some snapshots of an owl performing a perched landing, extracted from a reputed BBC documentary called "Life of Birds." The 
Table 1. Longitudinal and Lateral-Directional Variables

\begin{tabular}{|c|l|}
\hline Longitudinal Variables & \multicolumn{1}{|c|}{ Definition } \\
\hline$L, D, M$ & lift, drag and pitching moment \\
$q$ & pitch rate \\
$V_{\infty}$ & flight speed \\
{$\left[u_{B}, w_{B}\right]$} & $x_{B}$ and $z_{B}$ components of $V_{\infty}$ vector \\
$\alpha=\tan ^{-1}\left(w_{B} / u_{B}\right)$ & angle of attack \\
$\gamma$ & flight path angle \\
\hline \hline Lateral-Directional Variables & \multicolumn{1}{|c|}{ Definition } \\
\hline$N$ & yawing moment \\
$p, r$ & roll rate, yaw rate \\
$v_{B}$ & $y_{B}$ component of $V_{\infty}$, the flight speed \\
$\beta=\sin ^{-1}\left(v_{B} / V_{\infty}\right)$ & angle of sideslip \\
$\chi$ & wind axis heading angle \\
\hline
\end{tabular}

time histories of the flight path angle $\gamma$ (the angle made by the velocity vector in Fig. 2 with the horizontal plane), the body axis pitch angle $\theta$ (the angle made by the $x$ axis in Fig. 2 and the horizontal plane), and the angle of attack $\alpha$, obtained using vision processing tools in Matlab, are shown in Fig. 3.

The perching maneuver in the snapshots consists of two phases: (a) a gliding phase to bring the owl to a suitable position with respect to the landing spot, and (b) a rapid pitch up (usually to a post-stall angle of attack) accompanied by an instantaneous climb and rapid deceleration. The two-stage perching maneuver described here is representative of the perching maneuver used by the robotic aircraft in this paper (see Fig. 1a), as well as in the literature. Although the perching maneuver essentially involves controlling longitudinal parameters $\left(V_{\infty}\right.$ and $\gamma$ ), the control of lateral-directional dynamics cannot be ignored. The aerial robot considered in this paper, like the owl in Fig. 3, lacks a vertical tail, and the lateral-directional dynamics are therefore unstable.

\section{Literature Review}

A perching maneuver was studied analytically in [8], with the physically intuitive conclusion that a simple pitch-up, with the elevator deflected upwards to the fullest possible extent, is sufficient to achieve the rapid deceleration and flattening of the flight path required for perching. In other words, the profile shown in Fig. 3 was recovered analytically. It was demonstrated in [9] that perching aircraft do not lose their controllability even at the low flight speeds achieved during perching, and are consequently able to reject gusts and disturbances. Improvements resulting from the use of variable wing twist and movable tail boom were studied in $[10,11]$. In our prior work, ${ }^{12}$ a Linear Quadratic Gaussian (LQG) controller was designed for executing a perching maneuver using a combination of wing twist and wing dihedral (see Fig. 2) as control inputs.

An optimized perching maneuver was demonstrated in [13] on a robotic glider. Interestingly, the optimized strategy yielded a maneuver profile similar to that seen in Fig. 3: a "nominal" glide followed by a pitch-up with maximum upward elevator deflection. Recently, perching was demonstrated on a robotic aircraft which used spines attached to its legs to attach itself to a wall following a pitch up to a vertical attitude. ${ }^{14}$ The region of attraction for ensuring a successful perching maneuver after incorporating the dynamics and constraints of the attachment mechanism was computed in [15]. In [16,17], the authors experimentally demonstrated the feasibility of using wing articulation, particularly the wing dihedral angles, for controlling perching maneuvers.

The perching maneuver considered in the literature ${ }^{8-11,13,14}$ was strictly longitudinal, and the aircraft considered therein were laterally and directionally stable. Consequently, they ignored the lateral-directional motion of the aircraft (see Table 1). While it is true that a perching maneuver fundamentally involves controlling longitudinal flight parameters, viz., the speed $\left(V_{\infty}\right)$ and the flight path angle $(\gamma)$, the success of the maneuver can be severely impeded by the lateral-directional motion, particularly when the perched landing has to be accomplished on a small surface such as an electric pole or a human palm. The need 


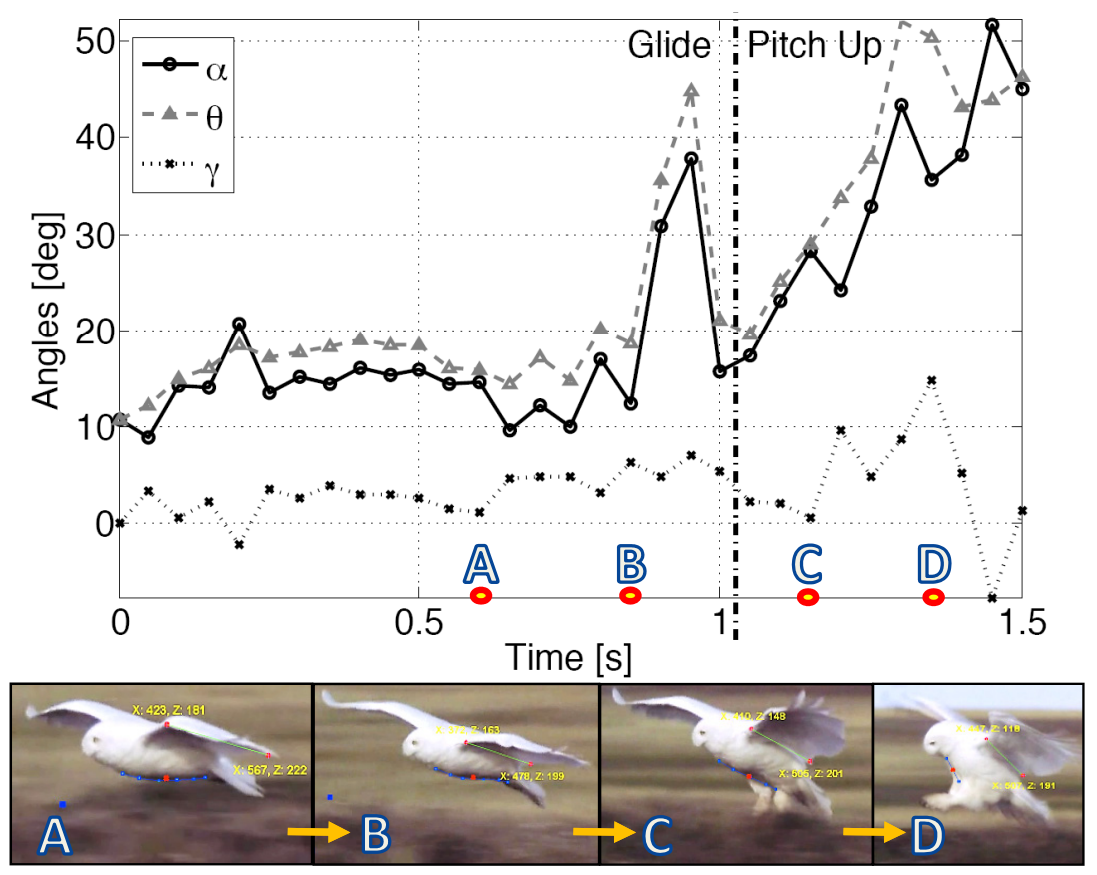

Figure 3. The angle of attack, flight path angle and pitch angle of an owl during a perching maneuver. The snapshots show the owl in various stages of the maneuver. The maneuver consists of two phases: a gliding phase (almost horizontal in this case) and a rapid pitch up to a high angle of attack. Video credits: The Life of Birds, produced by BBC Natural History Unit and PBS.

for controlling the lateral-directional motion becomes critical if the aircraft lacks a vertical tail, as birds do, in which case, the lateral-directional dynamics are highly unstable with a time constant that matches the duration of a typical perching maneuver. ${ }^{16,18}$ In the absence of a vertical tail, wing articulation is a promising capability which can be used for both longitudinal and lateral-directional control. ${ }^{18}$

The experiments in [16] demonstrated the feasibility of using the wing dihedral for longitudinal and lateral-directional flight control, and covered the two key elements of perching: control of flight path $(\gamma)$ with controlled lateral-directional control, and the pitch up. The theoretical foundations for the aircraft concept were laid by our prior work. ${ }^{18}$ We showed that (a) the wing dihedral can be varied symmetrically, along with the horizontal tail, to control the flight path angle independently of the flight speed, which is impossible in traditional fixed wing aircraft which use the horizontal tail setting as the only longitudinal control input; (b) the wing dihedral angles can be set asymmetrically for executing rapid turns, and (c) the sign of the yaw moment derivative with respect to anti-symmetric dihedral depends strongly on the angle of attack and angular rates. In [16], we suggested that trailing edge flaps on the articulated segments of the wing could be used to ensure uniform yaw control authority from the wing dihedral across the routine flight envelope.

\section{Objectives and Contributions}

The primary objective of this paper is to prove the practical viability of using wing dihedral (i.e., the "flapping" motion) for longitudinal as well as lateral-directional control during flight maneuvers, particularly perching. A perching maneuver requires three key ingredients: (a) a guidance law which brings the aircraft to a desired position, (b) a controller which regulates the heading, and (c) identification of a suitable point to commence the pitch-up to reduce the touchdown speed. In particular, Task (b) has been largely ignored in the literature. Task (c) has been addressed in [16], although its usefulness is contingent on accomplishing Tasks (a) and (b). In this paper, we focus on Tasks (a) and (b).

The contributions of this paper are as follows.

1. This paper demonstrates controlled perching of a laterally unstable aircraft controlled by articulated wings. The MAV autonomously performs a perched landing on a target object while simultaneously actively stabilizing and controlling its lateral-directional motion. 
2. Guidance algorithms are derived for the flight path angle and heading, whose dynamics are obtained by rewriting the flight dynamic equations in the spatial domain rather than as functions of time, after which dynamic inversion is employed. ${ }^{19-21}$ The dynamic inversion-based controller can be simplified to a (spatial) proportional-integral-derivative (PID) controller The stability of the controllers is proven rigorously.

3. The problem of non-uniform sign of the yaw control effectiveness of anti-symmetric dihedral was identified in [18], while [16] proposed a trailing edge flap-based approach to overcome it. This paper experimentally validates the proposition in [16], which is a unique application of trailing edge flaps for control.

This paper is organized as follows. The background material in flight dynamics with articulated wings ${ }^{16,18}$ is presented in Section II. The control law design has been described in Section III. It is shown that nonlinear dynamic inversion naturally leads to equivalent PID controllers, with exact gain tuning rules. The new perching control laws, successfully tested in the experiments, are described in Section IV. Results of successful perching experimentation are given in Section V.

\section{Flight Mechanics of Wing Articulation}

In this section, we will briefly review the theoretical underpinnings of the utility of wing dihedral for longitudinal and lateral-directional control. ${ }^{18}$ Thereafter, a novel scheme based on flaps is presented to overcome the controllability problems that arise from the use of wing dihedral for yaw control. Finally, we present the equations of motion and review the dynamics, which serves as a prelude to the discussion on control design in the following sections.

\section{A. Using the Wing Dihedral Angles for Control}

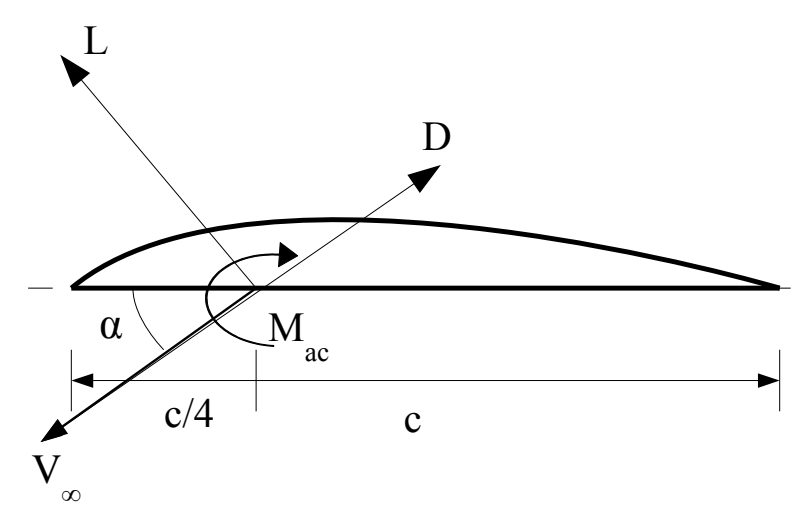

Figure 4. Forces and moments on a wing cross section. The lift and drag act perpendicular and along the wind velocity $V_{\infty}$, respectively, and in the plane of the airfoil. The term $M_{\mathrm{ac}}$ denotes the quarter chord pitching moment which does not depend on the angle of attack.

Figure 4 shows the forces acting on a wing section. The lift and drag both act in the plane of the airfoil; the lift is perpendicular to the local wind velocity, while drag acts along the local wind velocity. The quarter-chord pitching moment, $M_{\mathrm{ac}}$, is produced as a result of the pressure distribution on the airfoil and is independent of the angle of attack $\alpha$. Lift, drag and the quarter-chord pitching moment, all of them per unit wing span, are written as follows: ${ }^{22}$

$$
\begin{aligned}
L & =\frac{1}{2} \rho V_{\infty}^{2} c C_{L}(\alpha), \\
D & =\frac{1}{2} \rho V_{\infty}^{2} c C_{D}(\alpha), \\
M_{\mathrm{ac}} & =\frac{1}{2} \rho V_{\infty}^{2} c^{2} C_{m, a c}
\end{aligned}
$$




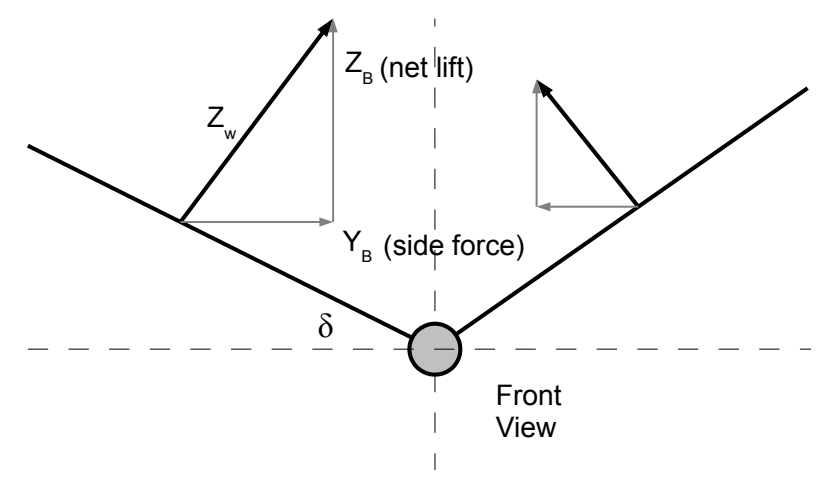

Figure 5. Illustration of the physics underlying the use of dihedral as a control. This picture shows a front view of the aircraft. The dihedral angle, $\delta$, is the complement of the angle between the plane of the wing and the plane of symmetry of the aircraft, and it is positive when the wing is deflected upwards in the local body frame.

where $c$ denotes the chord length (see Fig. 4), and the non-dimensional numbers $C_{L}(\alpha), C_{D}(\alpha)$ and $C_{m, a c}$ are called coefficients of lift, drag, and quarter chord pitching moment, respectively.

Figure 5 illustrates the physics underlying the use of wing dihedral as a control. The key point is that changing the wing dihedral re-orients the lift vector with respect aircraft $z$-axis. Increasing the wing dihedral reduces the force acting in the body $z$-direction, and generates a side force. The reduced $z$-force manifests in the form of reduction in the net lift acting on the aircraft, which is accompanied by an incommensurate reduction in the drag force. Thus, changing the wing dihedral angle alters the lift-to-drag (L/D) ratio of the aircraft and offers the option of controlling the flight path angle and the aircraft speed independently of each other. Figure 6 shows the flight path angle as a function of the symmetric wing dihedral deflection. The points shown in the figure are equilibria computed at the same flight speed of $3 \mathrm{~m} / \mathrm{s}$.

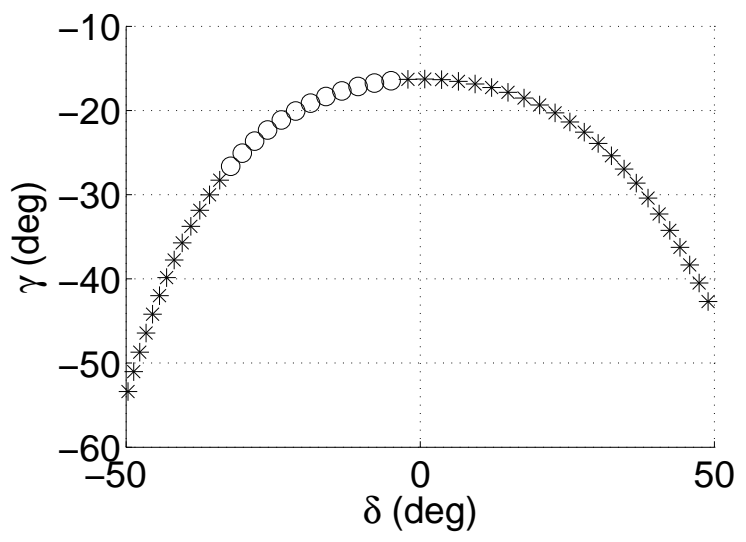

Figure 6. Bifurcation diagram, from, ${ }^{18}$ showing the steady state flight path angle as a function of the symmetric dihedral wing deflection. All equilibria are computed for a flight speed of $3 \mathrm{~m} / \mathrm{s}$. The shape of the markers denotes the qualitative nature of stability and should be ignored for the purpose of this paper.

On the other hand, the side force can be used for providing the centripetal force for turning, and as a source of yawing moment. In particular, if the CG is located behind the line of action of the side force, then a positive (rightward) side force produces a positive yawing moment and vice-versa. It follows that a positive rolling moment (wherein the lift on the left wing is higher than the right wing) is accompanied by a positive yawing moment if the wings have a positive dihedral deflection. In aircraft which lack a vertical tail, a positive rolling moment is accompanied by a negative yawing moment which leads to an increase in sideslip, with the consequence that the aircraft faces an increased drag and potential reduction in lateral-directional stability. This yawing moment which acts in a sense opposite to the rolling motion is called adverse yaw. Adverse yaw is one of the primary limiting factors for the lateral-directional performance of a vertical-tailless aircraft, and a positive wing dihedral naturally suppresses adverse yaw produced due to rolling. 


\section{B. Use of Trailing Edge Flaps for Mitigating Control Effectiveness Problems}

It was shown in [18] that yaw control effectiveness of the anti-symmetric dihedral $\left(\delta_{\text {asym }}\right)$ depends not only on the angle of attack, but also on the angular rates. The non-dimensional control effectiveness is given by

$$
N_{\delta_{\text {asym }}}=\left(x_{a} C_{L}+C_{m, a c}\right)
$$

where $C_{L}$ and $C_{m, a c}$ are the coefficients of lift and quarter chord pitching moment, introduced in (1). The term $x_{a}$ denotes the non-dimensional (with respect to chord length) distance between the center of gravity and the quarter-chord line. Therefore,

$$
\operatorname{sign}\left(N_{\delta_{\text {asym }}}\right)=\operatorname{sign}\left(x_{a} C_{L}+C_{m, a c}\right)
$$

For positively cambered wings, $C_{m, a c}<0$, and therefore, at small angles of attack, where $C_{L}$ is small, the control effectiveness is negative. At higher angles of attack, the control effectiveness is positive. For an intermediate range of angles of attack, the sign depends on the angular rates as well, as shown in Fig. 7. This can cause problems for yaw control, particularly when the angle of attack varies across the three regions in the course of a maneuver.

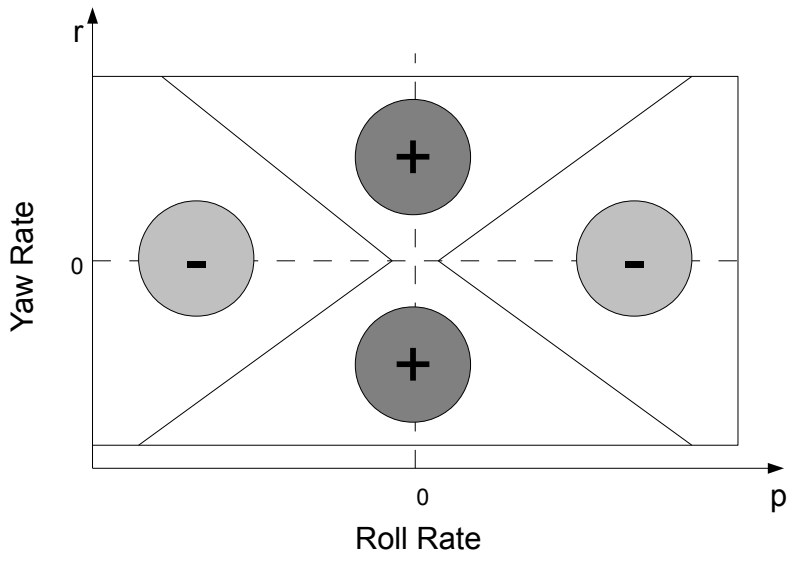

Figure 7. The yaw control effectiveness measure, $\operatorname{sign}\left(N_{\delta_{\text {asym }}}\right)$, as a function of the roll rate, $p$, and yaw rate, $r$.

One way to address the problem of non-uniform sign of control effectiveness is to use trailing edge flaps. Trailing flap deflection leads to a greater increase in $C_{L}$ as compared to the reduction in $C_{m, a c}$. Using thin airfoil theory, ${ }^{22}$ it can be shown that the change in $C_{L}$ and $C_{m, a c}$ due to a flap deflection $\delta_{f}$ is given by

$$
\begin{aligned}
& \Delta C_{L}=\left(2\left(\pi-\theta_{f}\right)+2 \sin \theta_{f}\right) \delta_{f} \\
& \Delta C_{m, a c}=-\frac{\delta}{2} \sin \theta_{f} \cos \left(\theta_{f}-1\right),
\end{aligned}
$$

where $\theta_{f} \in[0, \pi]$ depends on the location of the flap $\left(x_{f} c\right)$ from the leading edge:

$$
\cos \theta_{f}=1-2 x_{f}
$$

The term $\theta_{f}$ is defined purely for mathematical convenience in thin airfoil theory. For the aircraft considered in this paper, shown in Fig. $12, x_{f} \approx 0.8$ and $x_{a}=0.25$. Thus, $\theta_{f}=2.2143, \Delta C_{L}=3.45 \delta_{f}$ and $\Delta C_{m, a c}=$ $-0.14 \delta_{f}$.

It is of interest to find the flap deflection, as a function of $\alpha$, which will guarantee a certain positive control effectiveness. For example, suppose we need the effectiveness to be at least 0.025 (corresponds to an $\alpha$ of 10 deg in Fig. 8). Then, substituting the expressions for $\Delta C_{L}$ and $\Delta C_{m, a c}$, it follows that

$$
\begin{aligned}
& \frac{C_{L}}{4}+C_{m, a c}+0.72 \delta_{f}=0.025 \\
\therefore \quad & 0.07+0.5 \alpha-0.1311+0.72 \delta_{f}=0.025 \\
& \Longrightarrow \delta_{f}=0.12-0.69 \alpha
\end{aligned}
$$




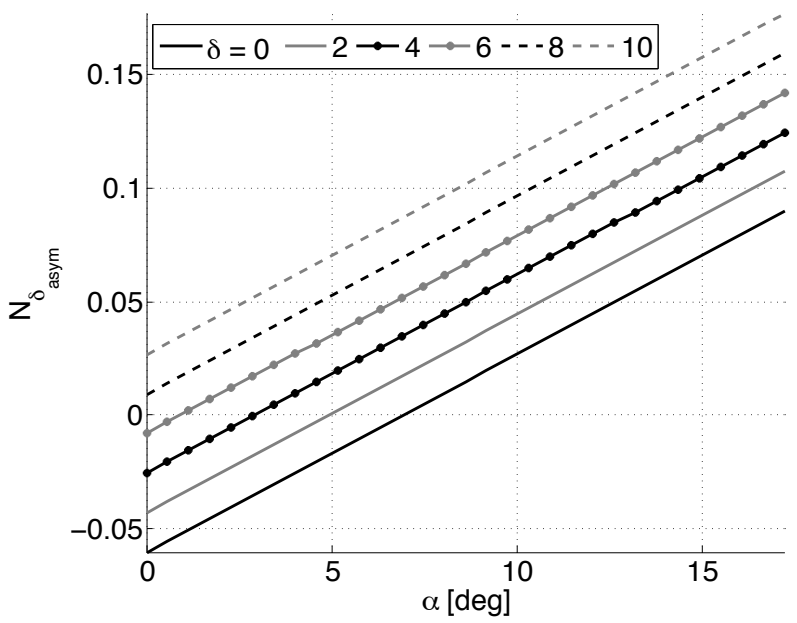

Figure 8. Effect of using a trailing edge flap, $0 \leq \delta_{f} \leq 10 \mathrm{deg}$, where the nondimensional control effectiveness, $N_{\delta_{\text {asym }}}$, is defined in (2).

Thus, flap deflection of nearly $7 \mathrm{deg}$ is required at $\alpha=0$, and no flap deflection is required beyond $\alpha=10$ deg.

The benefit of a uniformly positive control effectiveness, however, comes at a price: the aircraft is forced to fly in a high-lift (it can be checked that $C_{L}>0.64$ ), high drag configuration across the flight envelope. This necessarily means that the aircraft will fly slower than it otherwise would. However, note that the flight path angle can still be controlled effectively using symmetric dihedral deflection.

\section{Equations of Motion of Articulated-Winged Robots}

The rigid flight dynamics, together with the aerodynamics and kinematics, are highly nonlinear. The equations of motion, ignoring terms that arise from the angular velocity of the wing motion (due to flapping), have essentially the following structure: ${ }^{18,23}$

$$
\begin{array}{r}
m\left(\dot{\mathbf{u}}_{B}+S\left(\boldsymbol{\omega}_{B}\right) \mathbf{u}_{B}+\left(S\left(\dot{\boldsymbol{\omega}}_{B}\right)+S^{2}\left(\boldsymbol{\omega}_{B}\right)\right) \mathbf{r}_{c g}\right)=\mathbf{F}_{\text {net }} \\
\mathbf{J} \dot{\boldsymbol{\omega}}_{B}+S\left(\boldsymbol{\omega}_{B}\right) \mathbf{J} \boldsymbol{\omega}_{B}+m\left(S\left(\mathbf{r}_{c g}\right) \dot{\mathbf{u}}_{B}\right. \\
\left.+S\left(\boldsymbol{\omega}_{B}\right) S\left(\mathbf{r}_{c g}\right) \mathbf{u}_{B}\right)=\mathbf{M}_{\text {net }}
\end{array}
$$

where $m$ is the total mass of the aircraft, $m_{w}$ is the mass of each wing, $\mathbf{J}$ is the moment of inertia tensor for the aircraft, $S(\cdot)$ denotes a vector product, and $\mathbf{F}_{\text {net }}$ and $\mathbf{M}_{\text {net }}$ represent the net external (aerodynamic + gravitational) force and moment on the aircraft. Furthermore, $\boldsymbol{\omega}_{B}=[p, q, r]^{\mathrm{T}}$ is the vector representation of the aircraft angular velocity of the aircraft, with components in the aircraft body axes. The net aerodynamic force depends on the wing orientation, as discussed in Section A. The position of the aircraft center of gravity is denoted by $\mathbf{r}_{c g}$ which is, in turn, approximated closely by

$$
\mathbf{r}_{c g}=\frac{m_{w} b}{4 m}\left[0,\left(\sin \delta_{L}+\sin \delta_{R}\right),\left(\cos \delta_{L}-\cos \delta_{R}\right)\right]^{\mathrm{T}}
$$

where $\delta_{L}$ and $\delta_{R}$ are the dihedral angles of the left and right wings, and $b$ is the total wing span (so that each wing has length $b / 2$ ).

The force and moment vectors $\left(\mathbf{F}_{\text {net }}\right.$ and $\left.\mathbf{M}_{\text {net }}\right)$ depend strongly on the dihedral angles of the wings. In order to appreciate this point, we note, for example, that the yawing moment component of $\mathbf{M}_{\text {net }}$ is given by $^{18}$

$$
N=Z_{w, L}\left(\alpha_{L}\right) \sin \delta_{L}-Z_{w, R}\left(\alpha_{R}\right) \sin \delta_{R}
$$

where $\alpha_{L}$ and $\alpha_{R}$ are local angles of attack of the left and right wings (which actually vary as a function of $y$, the spanwise coordinate), and $Z_{w, L}$ and $Z_{w, R}$ are the local $z$-forces on the two wings (see Fig. 5). The terms $\alpha_{L}$ and $\alpha_{R}$ themselves depend on $\delta_{L}$ and $\delta_{R}$, respectively. For example, $\alpha_{R}$ at a spanwise coordinate 
$y$ is given by ${ }^{18}$

$$
\begin{aligned}
\alpha_{R} \approx & \beta \sin \delta_{R}+\alpha \cos \delta_{R}+\frac{p y+r x_{a} c \sin \delta_{R}}{u} \\
& +\frac{r y}{u} \alpha \cos \delta_{R}+\frac{p r y^{2} \cos \delta_{R}}{u^{2}}
\end{aligned}
$$

where $x_{a} c$ was defined in Section B as the distance between the center of gravity and the aerodynamic center of the wing. The sideslip $\beta$, roll rate $p$, and yaw rate $r$ were defined in Fig. 2. A similar expression can be written for $\alpha_{L}$.

In the simplest case, $Z_{w, L}$ and $Z_{w, R}$ are linear functions of $\alpha_{L}$ and $\alpha_{R}$, respectively. Even then, the flight dynamics of aircraft with articulated wings are nonlinear and non-affine-in-control. However, it is possible to use some knowledge about the flight dynamics to simplify control design:

1. The pitch dynamics can be controlled entirely by the elevator, and almost always independently of the lateral-directional dynamics. This is true for most aircraft, except those that lack a horizontal tail.

2. For our aerial robot, the pitch dynamics $(q, \alpha)$ are stable. The only source of instability is the lateraldirectionals dynamics involving the yaw rate $r$ and sideslip $\beta .{ }^{18}$

3. The roll dynamics are stable, and they are not controlled directly, since the wing dihedral primarily controls yaw with very little roll control effectiveness.

Next, we will formulate the control laws.

\section{Stability Theorems for Control Law Design}

Consider the problem of controlling the yaw ( $\beta$ and $r$ ) dynamics. For the purpose of controlling the yaw dynamics, (6) can be cast into the following control non-affine form

$$
\begin{aligned}
\dot{\eta}(t) & =f(t, \eta(t), \kappa(t), u(t)) \\
\dot{\kappa}(t) & =\zeta(\eta(t), \kappa(t), u(t), p(t))
\end{aligned}
$$

where $\eta(t)=(\beta(t), r(t)) \in \mathbb{R}^{2}$ represents the yaw dynamics, while $u \in \mathbb{R}$ is the control input, viz., the asymmetric dihedral deflection $\left(\delta_{\text {asym }}\right)$. The term $p(t)$ represents other control inputs, namely the elevator deflection $\left(\delta_{e}\right)$ and symmetric dihedral deflection $\left(\delta_{\mathrm{sym}}\right)$ which are used for longitudinal flight control. Finally, $\kappa \in \mathbb{R}^{6}=\left[V_{\infty}, \alpha, p, q, \theta, \phi\right]$ represents the rolling and pitching motion, as well as translation in the plane of symmetry (see Fig. 2). The flight dynamic modes corresponding to these six states are to be stable. ${ }^{18}$ One of the control objectives is to stabilize $\eta=(\beta(t), r(t))$ in (9), and ensure that it tracks a desired trajectory.

\section{A. Dynamic Inversion and PI(D) Control}

In this section, we will show that a class of dynamic inversion control laws, derived in [21], can be simplified into traditional proportional-integral (PI) or proportional-integral-derivative (PID) controllers. Moreover, the process of simplification yields exact gain tuning laws, which allows the control gains to be linked explicitly to the convergence properties of the closed loop system as well as the tracking error bound.

Consider a general system described by (9), where $\eta$ is no longer the yaw dynamics, but represents the state variables of interest for the purpose for control design. For now, we impose the additional condition $\eta \in D_{\eta} \subset \mathbb{R}$, where $D_{\eta}$ is compact. We will consider the case $\eta \in \mathbb{R}^{2}$ later in the section. Let $e(t)=\eta(t)-r(t)$ be the tracking error signal. Then, the open loop error dynamics are given by

$$
\begin{aligned}
& \dot{e}(t)=f(e(t)+r(t), \kappa(t), u(t))-\dot{r}(t), \quad e(0)=e_{0} \\
& \dot{\kappa}(t)=\zeta(e(t)+r(t), \kappa(t), u(t)), \quad z(0)=z_{0}
\end{aligned}
$$

where the unperturbed additional dynamics $\dot{\kappa}(t)=\zeta(0, \kappa(t), 0)$ are assumed to be exponentially stabilized by to the control input $p(t)$ (see $(9)$ ). We construct the dynamic inversion controller:

$$
\epsilon \dot{u}(t)=-\operatorname{sign}\left(\frac{\partial f}{\partial u}\right) \tilde{f}(t, \eta, \kappa, u)
$$


with $\epsilon>0$ sufficiently, and

$$
\tilde{f}(t, \eta, \kappa, u)=f(t, e+r, \kappa, u)-\dot{r}(t)+a_{m} e(t)
$$

and $a_{m}>0$ gives the desired rate of convergence of the closed loop dynamics.

Lemma 1. (Theorem 1, [21]) Given the system (10), the controller in (11) ensures that

1. the tracking error $e(t) \sim \mathcal{O}(\epsilon)$, and

2. the control $u(t)$ converges to the isolated root of $(12)$, i.e., $u(t)$ makes $f(\eta(t), \kappa(t), u(t)) \rightarrow\left(-a_{m} e(t)+\right.$ $\dot{r}(t))$. [21].

The proof of this theorem is based on Tikhonov's theorem ([24], Theorem 11.1), and may be found in

Remark 1. Although Lemma 1 guarantees that the tracking error $\sim \mathcal{O}(\epsilon)$, it does not provide any guarantees on robustness. In particular, using a high gain can be detrimental in the presence of time delays.

We now state the main result of this section.

Theorem 1. The control law in (11) is equivalent to a PI controller with proportional $\left(k_{p}\right)$ and integral $\left(k_{I}\right)$ gains tuned to satisfy $k_{p}=1 / \epsilon$ and $k_{i}=a_{m} k_{p}$, where $a_{m}$ is the desired time constant for the closed loop dynamics.

Proof: Since $\dot{\eta}=f(t, \eta, \kappa, u)$, and $\dot{e}=\dot{\eta}-\dot{r}$, we write the controller as

$$
\begin{aligned}
\epsilon \dot{u}(t) & =-\operatorname{sign}\left(\frac{\partial f}{\partial u}\right) \tilde{f}(t, \eta, \kappa, u) \\
& =-\operatorname{sign}\left(\frac{\partial f}{\partial u}\right)\left(\dot{e}(t)-a_{m} e(t)\right)
\end{aligned}
$$

Integrating both sides yields a PI controller of the form

$$
u(t)=u(0)-\operatorname{sign}\left(\frac{\partial f}{\partial u}\right) \frac{1}{\epsilon}\left(e(t)-e(0)-a_{m} \int_{0}^{t} e(t) d t\right)
$$

We may choose $u(0)=\operatorname{sign}(\partial f / \partial u) \epsilon e(0)$. If $k_{p}$ and $k_{i}$ denote the proportional and integral gains of the PI controller, then they should be chosen to satisfy

$$
k_{i}=a_{m} k_{p}, \text { and } k_{p}=1 / \epsilon
$$

so that

$$
u(t)=-\operatorname{sign}\left(\frac{\partial f}{\partial u}\right)\left(k_{p} e(t)+k_{I} \int_{0}^{t} e(t) d t\right)
$$

This completes the proof of Theorem 1.

Consider a second order system, $\ddot{\eta}(t)=f(t, \eta, \dot{\eta}, \kappa, u)$. We can write it in the form

$$
\begin{aligned}
& \dot{\eta}_{1}(t)=\eta_{2}(t) \\
& \dot{\eta}_{2}(t)=f_{2}\left(\eta_{1}, \eta_{2}, \kappa, t\right)+g_{2}(t) u(t)
\end{aligned}
$$

with $\eta=\eta_{1}$ as the output. The equation for $\dot{\eta}_{2}$ is affine in $u(t)$. The existence of $f_{2}(\cdot)$ and $g_{2}(\cdot)$ was shown in [25]. Note that $\operatorname{sign}\left(g_{2}\right)=\operatorname{sign}(\partial f / \partial \dot{\eta})$

Define the desired value of $\eta_{2}(t)$ as

$$
\eta_{2, d}(t)=-a\left(\eta_{1}(t)-r(t)\right)+\dot{r}(t)
$$

where $r(t)$ is the reference trajectory for $\eta(t)=\eta_{1}(t)$ and $a>0$. Define the error state for $\eta_{2}(t)$ as $e_{2}(t)=\eta_{2}(t)-\eta_{2, d}(t)$, whose dynamics are given by

$$
\dot{e}_{2}(t)=f_{2}\left(\eta_{1}, \eta_{2}, \kappa, t\right)+g_{2}(t) u(t)-\eta_{2, d}(t)
$$


From Theorem 1, the controller

$$
u(t)=-\operatorname{sign}\left(g_{2}\right)\left(k_{p} e_{2}(t)+k_{I} \int_{0}^{t} e_{2}(t) d t\right)
$$

ensures that $\eta_{2}$ tracks $\eta_{2, d}$, where the gains $k_{p}$ and $k_{I}$ are chosen as per the guidelines of Theorem 1 . It remains to simplify the controller to the PID form. Note that $e_{2}(t)=\eta_{2}(t)+a e_{1}(t)-\dot{r}(t)=\dot{e}_{1}(t)+a e_{1}(t)$. Substituting into (19), we get

$$
\begin{array}{r}
u(t)=-\operatorname{sign}\left(g_{2}\right)\left(k_{p} \dot{e}_{1}(t)+\left(a k_{p}+k_{I}\right) e_{1}(t)\right. \\
\left.+a k_{I} \int_{0}^{t} e_{1}(t) d t\right)
\end{array}
$$

which is a PID controller.

Theorem 2. The second-order system (16) can be stabilized using the PID controller (20), and moreover, it can be ensured that the tracking error between $\eta(t)$ and the reference signal $r(t)$ is bounded.

Proof: Theorem 1 guarantees that the control law (19) ensures that the tracking error $e_{2}(t)$ of the (18) is bounded. Thus, $\left\|\eta_{2}-\eta_{2, d}\right\|<\mathcal{O}(\epsilon)$ for some $\epsilon>0$. Consider now the first equation $\dot{\eta}(t)=\dot{\eta}_{1}(t)=\eta_{2}(t)$. Since $\eta_{2}(t)=e_{2}(t)+\eta_{2, d}(t)$, we can write

$$
\begin{aligned}
& \dot{\eta}(t)=-a(\eta(t)-r(t))+\dot{r}(t)+e_{2}(t) \\
\Longrightarrow \quad & \dot{e}_{1}(t) \triangleq \dot{\eta}(t)-\dot{r}(t)=-a e_{1}(t)+e_{2}(t)
\end{aligned}
$$

Since the unperturbed $e_{1}$ dynamics (obtained by setting $e_{2}=0$ ) are exponentially stable, it follows from the Comparison Lemma (Lemma 9.1 in [24]) that $e_{1}(t)$ is bounded if $e_{2}$ is bounded. Moreover, the bound on $e_{1} \sim \mathcal{O}(\epsilon)$. This completes the proof.

Remark 2. The following observations summarize the results in this section:

1. PI and PID controllers can be employed for nonlinear systems of the from (9) provided the additional ( $\kappa)$ dynamics are stable.

2. The dynamic inversion procedure yields a systematic gain tuning procedure ((15) and (20)).

\section{New Closed Loop Controller for Perching}

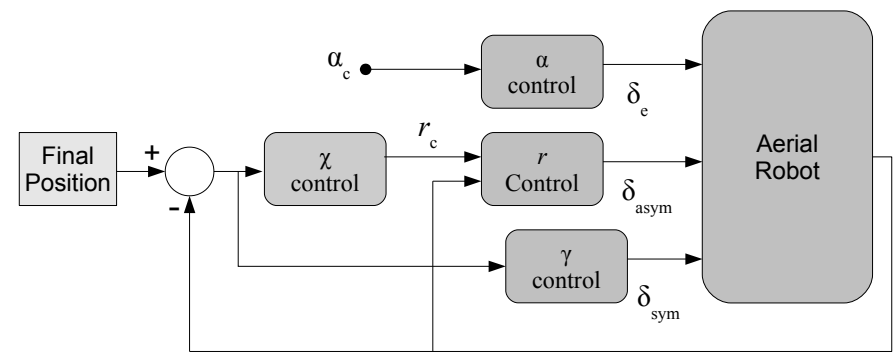

Figure 9. Controller block diagram for the aerial robot.

The objectives of controlled perching can be stated as follows:

1. Design a control law for the symmetric dihedral deflection $\left(\delta_{\mathrm{sym}}=\left(\delta_{L}+\delta_{R}\right) / 2\right)$ which ensures that the flight path $z(x)$ tracks the desired profile $z_{d}(x)$. The desired profile $z_{d}(x)$ is a straight line connecting the initial point to the desired final point $\left(x_{f}, z_{f}\right)$ (see Fig. 10).

2. Design a control law for the anti-symmetric dihedral deflection $\left(\delta_{\text {asym }}=\left(\delta_{L}-\delta_{R}\right) / 2\right)$ which ensures that $y(x) \rightarrow 0$ as $x \rightarrow x_{f}$, the desired final point. This objective requires that the yaw $(r)$ dynamics be stabilized. 
Additionally, the angle of attack is controlled by the elevator. The flaps are deflected to a constant angle of 10 deg. The controller block diagram is shown in Fig. 9. The control and path guidance algorithms in this section are new due to the unconventional control inputs $\delta_{\mathrm{sym}}$ and $\delta_{\text {asym }}$. The stability proofs are presented by rewriting the dynamical equations in the spatial domain rather than as functions of time, thereby permitting the use of dynamic inversion presented in Section III. The guidance problems have been illustrated schematically in Fig. 10.

The flight path angle $(\gamma)$ is given by ${ }^{18}$

$$
\begin{aligned}
\sin \gamma= & \cos \alpha \cos \beta \sin \theta-\sin \beta \sin \phi \cos \theta \\
& -\sin \alpha \cos \beta \cos \phi \cos \theta
\end{aligned}
$$

while the wind axis heading angle $\chi$ is calculated as follows

$$
\begin{aligned}
\sin \chi \cos \gamma= & \cos \alpha \cos \beta \cos \theta \sin \psi \\
& +\sin \beta(\sin \phi \sin \theta \sin \psi+\cos \phi \cos \psi) \\
& +\sin \alpha \cos \beta(\cos \phi \sin \theta \sin \psi \sin \phi \cos \psi)
\end{aligned}
$$

Yaw control has been often neglected in the literature on perching. On the other hand, lateral-directional control is an important concern for aircraft which lack a roll control surface, such as an aileron, and use a highly unconventional yaw control mechanism.

\section{A. Angle of Attack Control}

With a large horizontal tail, and the CG located approximately $c / 3$ behind the wing aerodynamic center, the aircraft was sufficiently stable in pitch. Given the excellent open loop stability characteristics, the angle of attack was not controlled by feedback laws. Rather, on the basis of open loop glide tests, the elevator deflection was set as a function of the commanded angle of attack:

$$
\delta_{e, c}=\frac{\pi}{108}\left(15-\alpha_{c}\right), \quad \ldots\left(\alpha_{c} \text { in degrees }\right)
$$

where $\delta_{e, c}$ and $\alpha$ have been specified in degrees instead of the more conventional units of radians.

\section{B. Control of Flight Path}

Consider the equation

$$
\dot{\gamma}=\frac{\rho S C_{L}}{2 m} V_{\infty} \cos \delta_{\mathrm{sym}}-\frac{g}{V_{\infty}} \cos \gamma
$$

where $m$ is the aircraft mass, $S$ is the wing area and $\delta_{\text {sym }}$ is the symmetric dihedral deflection $\left(\delta_{\mathrm{sym}}=\right.$ $\left.\left(\delta_{L}+\delta_{R}\right) / 2\right)$. Note that

$$
\frac{d \gamma}{d x}=\frac{\dot{\gamma}}{\dot{x}}=\frac{\dot{\gamma}}{V_{\infty} \cos \gamma}
$$

If we further assume that $\cos \gamma \approx 1$, then the equations of translational motion simplify to the following form:

$$
\begin{aligned}
& \frac{d z}{d x}=\tan (\gamma(x)) \\
& \frac{d \gamma}{d x}=\frac{\rho S C_{L}}{2 m} \cos ^{2} \delta_{\mathrm{sym}}(x)-\frac{g}{V_{\infty}^{2}}
\end{aligned}
$$

where $\delta_{\text {sym }}$ is the control input.

The system in (27) is in the form (16) except that derivatives and functions are defined with respect to $x$, not $t$. Hence, by replacing $t$ and $d t$ in (20) with $x$ and $d x$, the following controller is designed (see Theorem 2):

$$
\begin{array}{r}
\delta_{\mathrm{sym}}(x)=-\left(k_{p} \frac{d e_{z}}{d x}+\left(a k_{p}+k_{I}\right) e_{z}(x)\right. \\
\left.+a k_{I} \int_{0}^{x} e_{z}(x) d x\right)
\end{array}
$$



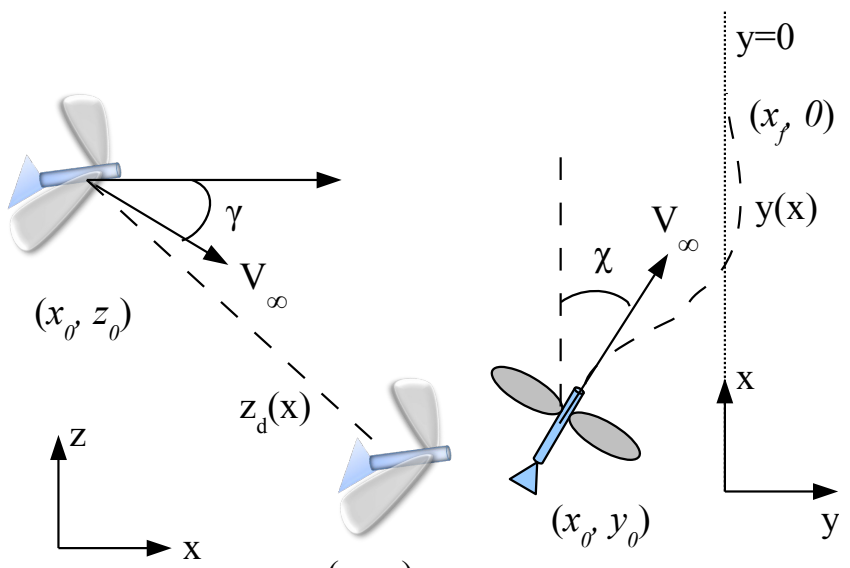

$\left(x_{f}, z\right)$

Figure 10. Schematic of the guidance problem. The aircraft is guided in the $x-z$ plane along $z_{d}(x)$, while the $y$ coordinate is regulated separately. Note that the $z$ axis points upwards. Consequently, the $x, y, z$ axes shown here do not define a conventional right-handed frame. This is purely a matter of convenience, and has no bearing on the calculations performed in the paper.

where $e_{z}(x)=z(x)-z_{d}(x)$, and $a$ is the desired rate of convergence of $z(x)$ to the desired trajectory $z_{d}(x)$. The above controller ensures that $z(x)$ tracks the commanded trajectory $\left(z_{d}(x)\right.$ in Fig. 10). The stability of the controller is guaranteed by Theorem 2. Note that the independent variable used in the controller is $x$, and not time $t$. This is consistent with the recasting of the equations of motion in the spatial domain.

\section{Control for Terminal Coordinates $(x, y)$}

The motion in the $x-y$ plane is given by

$$
\dot{x}=V_{\infty} \cos \gamma \cos \chi, \quad \dot{y}=V_{\infty} \cos \gamma \sin \chi
$$

We assume that the yaw rate, $r$, settles down rapidly to the commanded yaw rate $r_{c}$, so that $r_{c} \approx r=$ $\dot{\chi} / \cos \theta \cos \phi$. The inner loop yaw controller in Fig. 9, described in Section D, ensures that $r$ converges rapidly to $r_{c}$. This is an expression of the time scale separation between the fast yaw $(r)$ dynamics and the slower heading $(y, \chi)$ dynamics. Thus, we get

$$
\begin{aligned}
& \frac{d y}{d x}=\tan \chi(x) \\
& \frac{d \chi}{d x}=V_{\infty} \gamma \dot{\chi}(t)=\frac{V_{\infty} \cos \gamma}{\cos \theta \cos \phi} r_{c}(x)
\end{aligned}
$$

where $\phi$ is the bank angle of fuselage. Note that $\cos \gamma, \cos \theta$, and $\cos \phi$ are all positive since they lie in $[-\pi, \pi]$ (very generally), which implies that the control coefficient $V_{\infty} \cos \gamma /(\cos \theta \cos \phi)$ is uniformly positive. The control problem is very similar to what is encountered for the flight path angle, shown in (27), and we derive a similar controller as (28):

$$
\begin{gathered}
r_{c}(x)=-\left(k_{p} \tan (\chi(x))+\left(a k_{p}+k_{I}\right) y(x)\right. \\
\left.+a k_{I} \int_{0}^{x} y(x) d x\right)
\end{gathered}
$$

The stability of this controller is guaranteed by Theorem 2. Further, as in the case of flight path control, the use of dynamic inversion was considerably facilitated by rewriting the dynamical equations in the spatial domain which rendered them in a strict feedback form. Next, we describe the design of the inner yaw control loop in Fig. 10.

Remark 3. The choice of $y=0$ as the desired path can be replaced by any suitable path $y_{d}(x)$, such as a straight line connecting the initial point and the desired final point, as for the flight path angle guidance law. 


\section{Yaw Control}

The objective of the inner yaw control loop in Fig. 9 is to command the anti-symmetric wing dihedral, $\delta_{\text {asym }}$ so that the yaw rate $r$ tracks the yaw rate commanded by the outer loop, $r_{c}$, described in Section C. Yaw control is achieved using a PI controller, motivated by Theorem 1, given by

$$
\delta_{\text {asym }}=k_{p}\left(r_{c}(t)-r(t)\right)+k_{I} \int_{0}^{t}\left(r_{c}(t)-r(t)\right) d t
$$

The proportional and integral gains were set to $k_{p}=k_{I}=2$ during the experiments.

Remark 4. In practice, the heading and flight path angles are computed and commanded every $0.05 \mathrm{~s}$. This prevents undesirable oscillatory behavior which arises due to continuous changes in the wing dihedral, and the underlying coupling between the wing dihedral angle and the pitching dynamics of the aircraft. The control laws are effective over the short duration of the experiments, but may need to be improved for experiments which may last over a longer duration and require turning through large angles.

\section{E. Timing of the Pitch-Up}

The guidance laws derived guide the aircraft to a suitable point at which it pitches up in order to slow down for a perched landing. Optimization routines, such as those employed in $[8,13]$, can determine the timing of the pitch-up as part of the optimal trajectory calculations. In this paper, we use a simulation-based approach. We are specifically interested in determining the minimum information needed to determine the location of the pitch-up point so that the final position of the aircraft is within acceptable limits $(30 \mathrm{~cm}$ in our case) of the desired landing point.

It was found from a parameter study that it suffices to specify the point of pitch-up in terms of the $z$ coordinate (the altitude) of the aircraft. To measure the expected error radius, and verify the sanity of using the $z$-coordinate to command the pitch-up, we performed a series of numerical simulations on a longitudinal model of the MAV. Figure 11 shows the terminal speed $V_{f}$ and terminal $x$-coordinate $\left(x_{f}\right)$ at the time of landing for a range of initial flight speeds and flight path angles. The elevator is deflected to the maximum upward position when the aircraft is at $x=0$ and $z=0.8 \mathrm{~m}$. The $z$-coordinate of the landing point is given by $z=0$.

Figure 11 shows the terminal $x$ coordinate $x_{f}$ and terminal flight speed $V_{f}$ for different values of initial speed $V_{\text {in }}$ and initial flight path angle $\gamma_{\text {in }}$. These plots are $2-$ D projections of a $3-\mathrm{D}$ surface plot. First, from Fig. 11(a), we deduce that the aircraft lands at $z=0$ within a $30 \mathrm{~cm}$ error radius of $x_{f}=3 \mathrm{~m}$ when the initial flight speed is greater than $4.5 \mathrm{~m} / \mathrm{s}$. This is a reasonable scenario for our aircraft. Likewise, the red region of Fig. 11(b) confirms the value of the error bar across the entire range of $\gamma_{\text {in }}$. Moreover, from Fig. 11(c), we see that the terminal flight speed is actually minimized when the $V_{\text {in }} \approx 5 \mathrm{~m} / \mathrm{s}$. The terminal flight speed increases on either side of $V_{\text {in }}=5 \mathrm{~m} / \mathrm{s}$, and is a function of $\gamma_{\text {in }}$. Therefore, the guidance algorithm is required to bring the aircraft to point $3 \mathrm{~m}$ away and $0.8 \mathrm{~m}$ from the desired landing point.

\section{Results of Flight Tests}

We can envision the experimental perching maneuver in this section as being initiated after the flapping wing aerial robot stops wing flapping to perch on a target.

\section{A. Experimental Setup}

The experiments described in this paper were performed on a test aerial robot, shown in Fig. 12. Note that the aerial robot lacks a vertical tail. The original wing was cut to facilitate hinging of the outboard $60 \%$ of the wing. It has five control surfaces:

1. An elevator, which is a movable flap attached to the horizontal tail, and whose deflection is denoted by $\delta_{e}$.

2. The dihedral angles $\left(\delta_{R}\right.$ and $\left.\delta_{L}\right)$ of the outboard segments of the right and left wing can be changed independently of each other. The actuators for changing the wing dihedral angle were attached on the lower surface of the center (non-rotating) wing section, along with the radio receiver. 


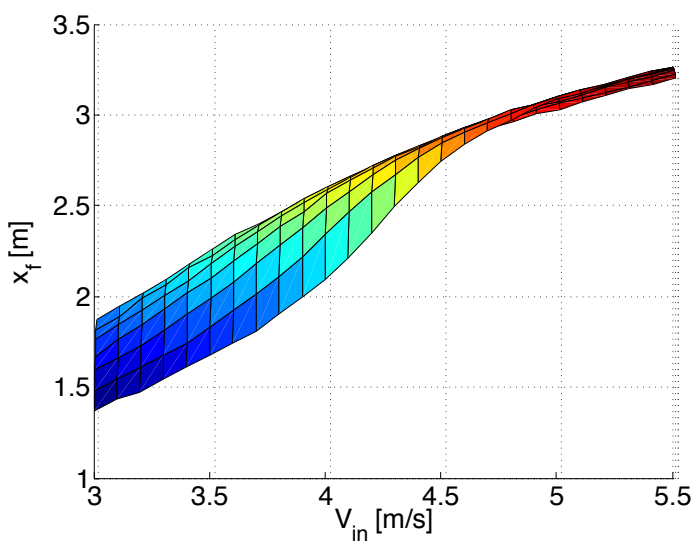

(a) $x_{f}$ versus $V_{\text {in }}$

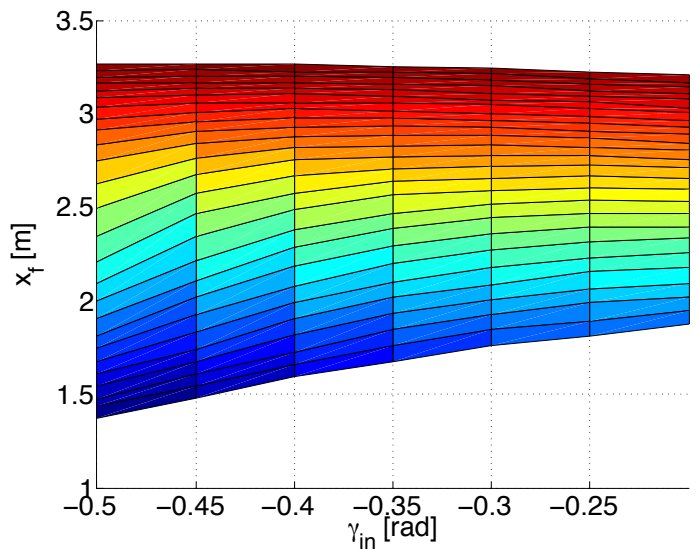

(b) $x_{f}$ versus $V_{\text {in }}$

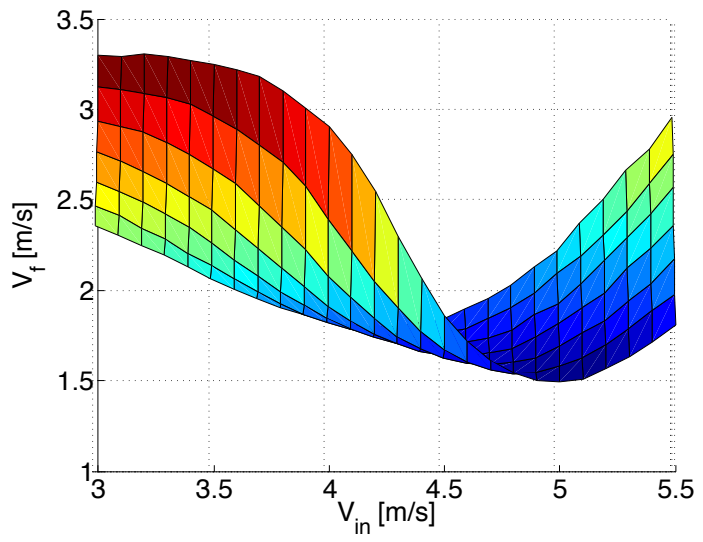

(c) $V_{f}$ versus $V_{\text {in }}$

Figure 11. 2D projections showing the variation of the terminal position $x_{f}$ and flight speed $V_{f}$ as functions of initial speed $V_{\text {in }}$ and flight path angle $\gamma_{\text {in }}$.

3. The outboard segments are equipped with flaps which are capable of being actuated independently. In our experiments, both flaps were deflected by the same amount $\delta_{f}$. Flaps are used for ensuring uniform yaw control effectiveness, as explained in Section B. Note that these flaps, when deflected in an anti-symmetric manner, can also act as the traditional ailerons.

The geometric properties for the MAV are listed in Table 2. Both wings can rotate from a maximum $45 \mathrm{deg}$ dihedral to minimum $-15 \mathrm{deg}$ for a total arc range of $60 \mathrm{deg}$. The actuators for wing dihedral, it may be recalled, are controlled independently on both wings. Digital actuators with a torque rating of $0.29 \mathrm{~kg}-\mathrm{cm}$ are used to maneuver the wings. The time required for the wings to rotate from the minimum -15 deg to maximum $45 \mathrm{deg}$ is about $0.05 \mathrm{~s}$, and the actuators have a time-delay of $200 \mathrm{~ms}$.

Flight data was measured using the Vicon motion-capture system. The system consists of 16 infrared cameras which track reflective markers attached to the various articulated parts of the robot. The Vicon system uses triangulation to locate the markers with an accuracy of $1 \mathrm{~mm}$. A recording rate of $100 \mathrm{~Hz}$ is used to capture the position and orientation data. In practice, however, the operating frequency is $60 \mathrm{~Hz}$ after allowing for offline computation, and control signals are transmitted at $20 \mathrm{~Hz}$.

The real-time data stream provided by the Vicon motion-capture system includes the global reference position and the orientation of each object (the fuselage and the two wings). The availability of tracking data is contingent upon the visibility of the objects. For time-steps with information loss, which were minimal 


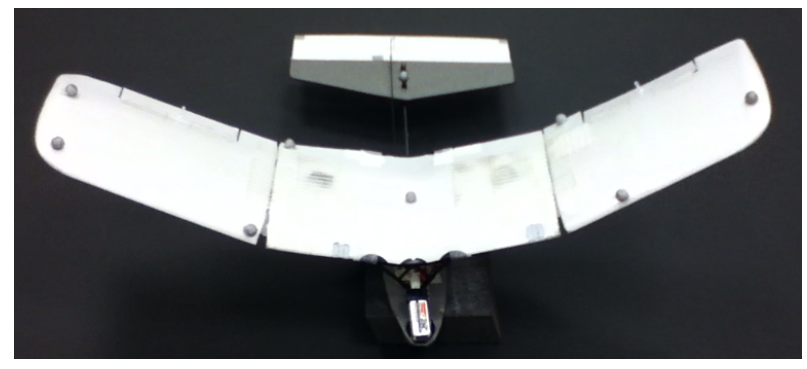

(a) Symmetric dihedral configuration

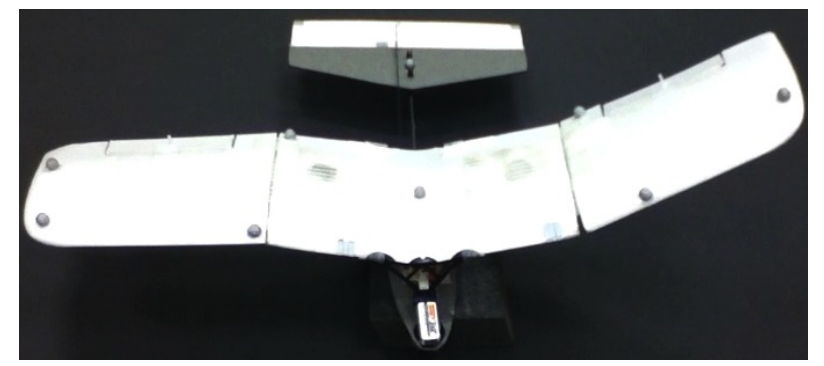

(b) Asymmetric dihedral configuration

Figure 12. The aerial robot, developed by the authors, is shown in symmetric and asymmetric dihedral wing configurations.

Table 2. Physical Properties of the MAV

\begin{tabular}{|l|c|c|}
\hline \hline Property & Metric Measurement & Units \\
\hline Mass $(m)$ & 44.0 & $\mathrm{~g}$ \\
Wing span $(S)$ & 41.8 & $\mathrm{~cm}$ \\
Wing chord $(c)$ & 9.5 & $\mathrm{~cm}$ \\
Wing incidence angle & 6.0 & $\mathrm{deg}$ \\
Wing dihedral (left and right) & controlled-variable & \\
MAV length & 35.0 & $\mathrm{~cm}$ \\
Elevator area & 39.12 & $\mathrm{~cm}^{2}$ \\
\hline \hline
\end{tabular}

and rarely comprised consecutive frames, a linear fit was used to estimate the missing data. Experiments were performed within the effective volume of capture, containing an area of $6 \mathrm{~m}$. $\times 4 \mathrm{~m}$. and a height of $2 \mathrm{~m}$. Since Vicon provides only position and attitude information, a second order Lagrangian polynomial was used to compute velocities and angular rates, which were then filtered to eliminate noise.

\section{B. Experimental Results}

The experiments consisted of a series of flight tests of the aerial robot. Each flight test started with a hand launch of the aerial robot from a height of approximately $2.5 \mathrm{~m}$. The flaps were deflected to $10 \mathrm{deg}$ in order to ensure yaw controllability, as explained in Section B. Control signals, were computed offline and transmitted to the robot only after it entered the field of view of the cameras. For experiments involving perched landing on the hand, the guidance laws were provided the $x-z$ coordinates of the point at which the pitch up was to be commenced. The pitch-up command consisted of deflecting the elevator to the maximum upward position while simultaneously setting the wing dihedral to zero. The command for executing the pitch-up was sent solely based on the altitude of the robot, as explained in Sec. IV-E, and to prevent unnecessarily hard landings.

The results described in this section were obtained during two flight tests which ended in a successful perched landing on a hand. A montage of snapshots taken from their video recordings are shown in Fig. 13. The flight parameters recorded during the tests are shown in Figs. 14 and 15. Each set shows the trajectory in the 3D space, and the time histories of the flight speed, angle of attack, sideslip and the flight path angle. The following observations can be made, which are common to both sets:

1. The entire maneuver lasts just $1.5 \mathrm{~s}$, which is close to the settling time of the closed loop dynamics. Only the fast dynamics stabilize entirely within this range. The success of the guidance loop, on the other hand, can be severely compromised if the initial heading offset from the desired path to the target is large. This is because the dynamics of translational motion have a time constant as the duration of the maneuver. 

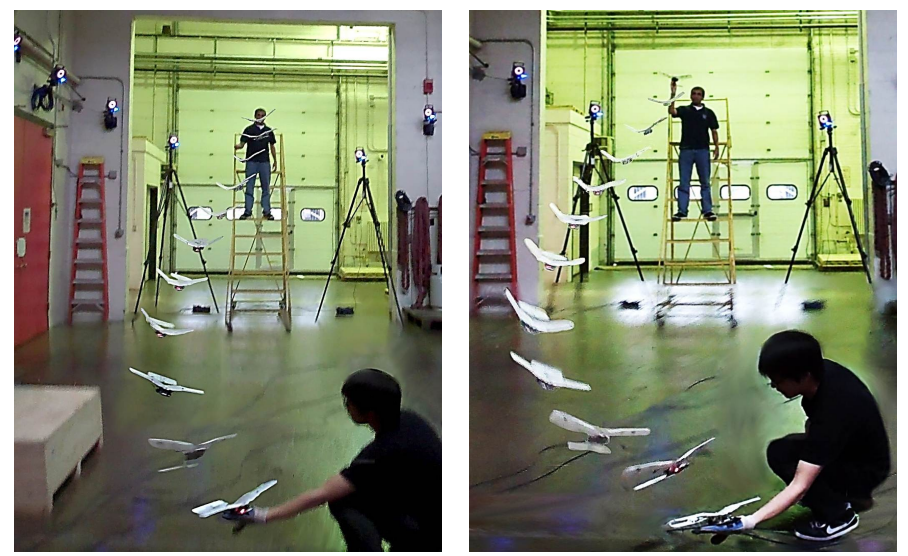

Figure 13. Montage of snapshots taken from the video of a flight test showing perching on a hand (a supplementary video has been submitted.)

2. The angle of attack settles down to a nominal value of approximately 10 deg quite rapidly during the gliding phase. Thereafter, it increases to a peak value around 50 deg during the pitch-up which terminates in a perched landing.

3. During the pitch-up phase, the flight path angle increases to around $-10 \mathrm{deg}$, and the flight speed drops significantly from a peak value of $4.7 \mathrm{~m} / \mathrm{s}$ to just under $2.5 \mathrm{~m} / \mathrm{s}$ at the time of landing, a reduction to nearly $50 \%$ of the original speed.

4. The sideslip is well regulated for the entire duration of the flight tests.

5. The maximum sideways offshoot of the trajectory (the maximum $y$ coordinate) is nearly $1 \mathrm{~m}$, but the guidance algorithms rapidly correct the course and bring the aircraft to an appropriate point for executing the pitch-up.

Clearly, we have demonstrated all the three elements of perching stated earlier: closed loop flight path control, control of the lateral-directional dynamics, and significant speed reduction following a rapid pitch up leading up to the landing.

\section{Experimental Observations and Pointers}

Due to the slow time scale of the translational dynamics, it is important to restrict the initial sideward deviation or heading offset. In our case, an initial sideward offset of $1 \mathrm{~m}$ (which amounts of $20 \%$ of the total length of the flight path) was compensated for by the controller, although it occasionally required aggressive maneuvering. This limitation is purely physical and arises due to the absence of thrust (which would have even allowed the aircraft to hover if required). It is not related to the design of the controllers.

The timing of the pitch-up command leading to the perched landing is critical, and some tuning is required to accommodate the actuator time delays. In our case, we sent the command when the aircraft was nearly $1.5 \mathrm{~m}$ above the ground. Interestingly enough, the experimentally observed error margins match well with the analytical predictions in Fig. 11.

Although dihedral-based yaw control is capable of turn rates as high as $100 \mathrm{deg} / \mathrm{s},{ }^{18}$ it is advisable to restrict the maximum commanded turn rate if ailerons are absent. When ailerons are absent, roll rate is produced purely in reaction to the yaw rate and sideslip. Consequently, a large sideslip is produced in the process of achieving a large turn rate. Since tailless aircraft lack directional stability, ${ }^{18}$ it takes a large control effort and time to stabilize the yaw dynamics about the commanded flight states. We restricted the maximum commanded turn rate to $30 \mathrm{deg} / \mathrm{s}$.

Finally, we noted in Section A that the actuator response shows a time delay of $0.2 \mathrm{~s}$. A substantial time delay is one of the factors that serve to set an upper limit on the control gains. Our choice of control gains was adequate to ensure good performance even in the presence of a substantial time delay. In general, a controller similar to ours would need to be checked for robustness to time delays on a case-by-case basis, 


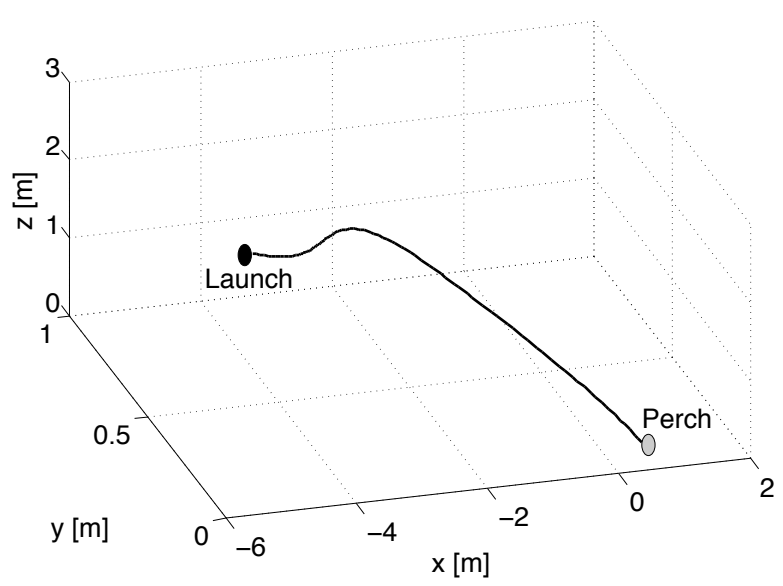

(a) $3 \mathrm{D}$ view of the robot trajectory

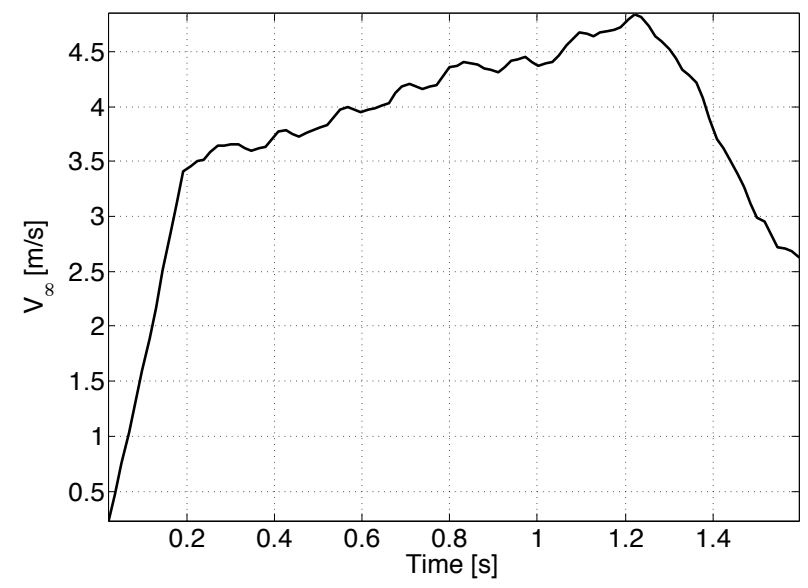

(b) Time history of the flight speed (filtered)

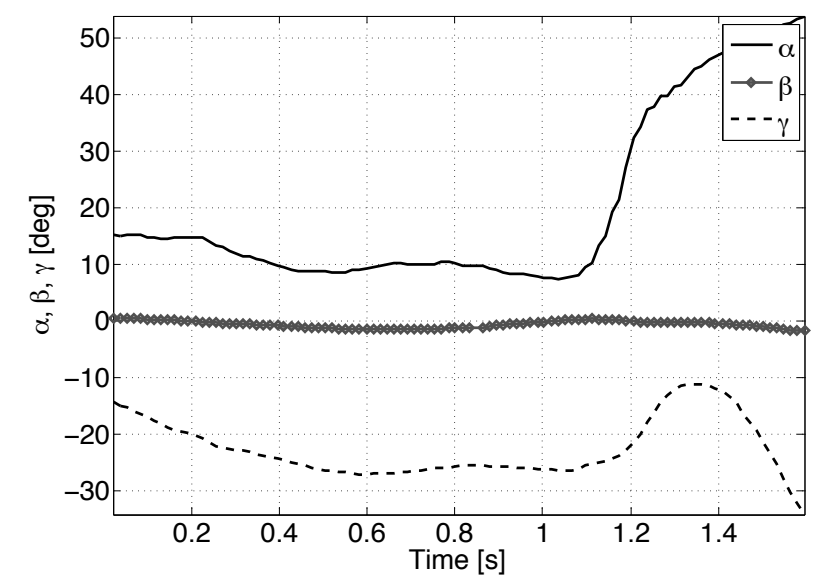

(c) Angle of attack $\alpha$, sideslip $\beta$, and flight path angle $\gamma$.

Figure 14. Flight parameters from an successful experiment of perching on a hand. 


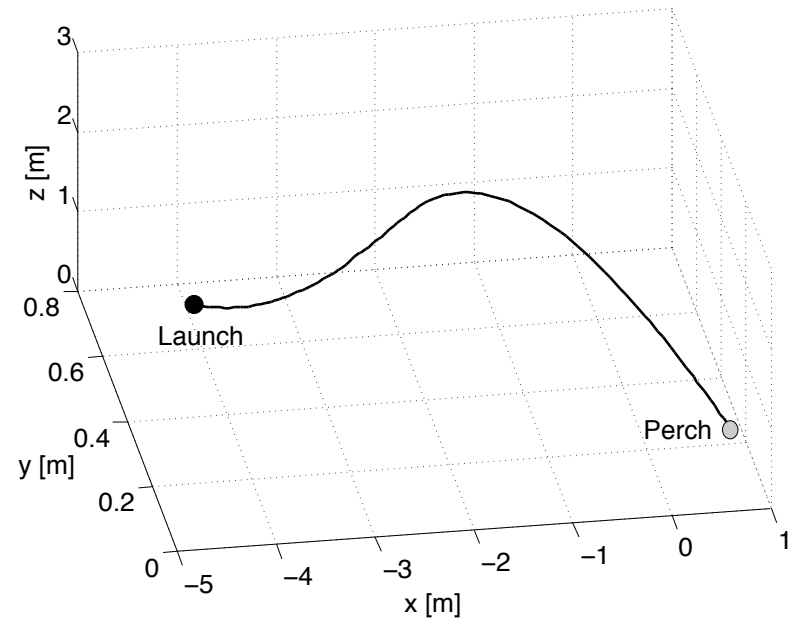

(a) 3D view of the robot trajectory

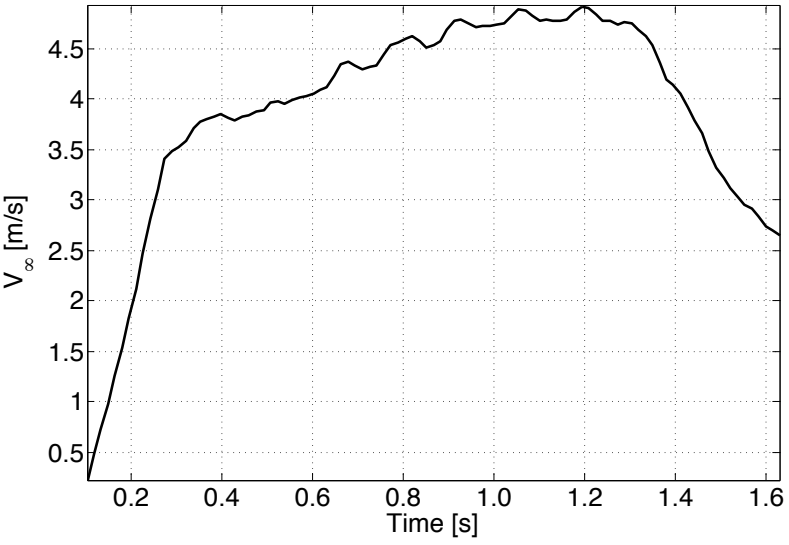

(b) Time history of the flight speed (filtered)

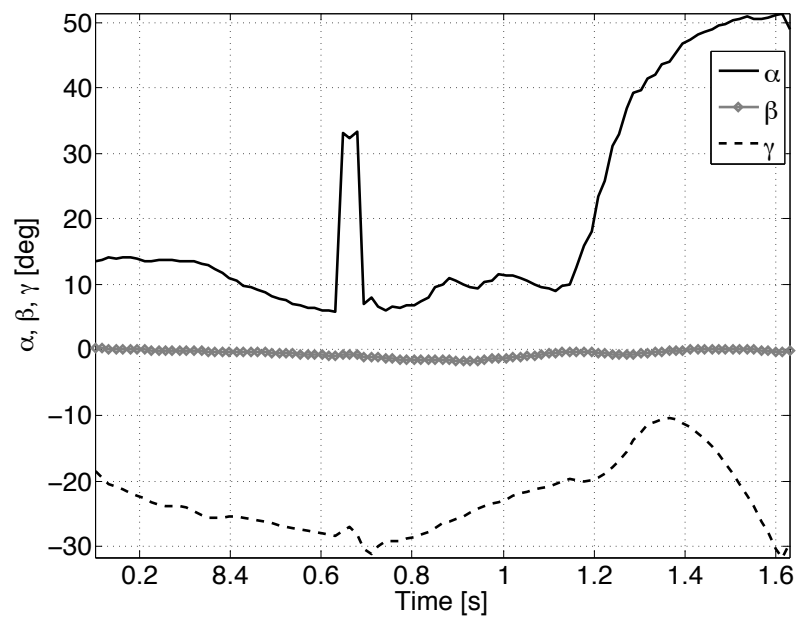

(c) Angle of attack $\alpha$, sideslip $\beta$, and flight path angle $\gamma$.

Figure 15. Flight parameters from another experiment of perching on a hand. The peak in $\alpha$ just after $0.6 \mathrm{~s}$ is the result of instantaneous measurement errors, which are filtered out by the controller. 
because robustness to time delays is not built into dynamic inversion by design (as compared to the approach adopted in [25]).

\section{Conclusion}

This paper reported the first perching demonstration on a laterally unstable aerial robot. The lateraldirectional motion of the aircraft was controlled actively for the entire duration of the maneuver. This complements and advances the state of the art experiments reported in the literature which were concerned almost exclusively with the longitudinal motion of stable aircraft. We used variable, asymmetric wing dihedral effectively to control the flight path as well as the heading of the aerial robot. Trailing edge flaps were used to ensure that the wing dihedral provided uniform yaw control effectiveness across the flight envelope. Novel closed loop guidance laws were designed for perching by rewriting the equations of motion in the spatial domain and applying dynamic inversion-motivated PID control. The hand perching demonstration represents a significant step in developing aerial robots capable of close operational interaction with humans. Future work should focus on refining the controller for large external disturbances, and a take-off and goaround capability to accommodate failures during perching attempts.

\section{Acknowledgments}

This project was supported by the Air Force Office of Scientific Research (AFOSR) under the Young Investigator Award Program (Grant No. FA95500910089) monitored by Dr. W. Larkin. The concept of wing articulation was presented to the authors by Dr. Gregg Abate at AFRL. Nihar Gandhi and Xichen Shi, both of them undergrad students, and Sana Ashraf, a graduate student at UIUC, helped build the aircraft and also participated in the experiments. Their contributions are gratefully acknowledged.

\section{References}

\footnotetext{
${ }^{1}$ AeroVironment, Inc. (AV): Nano Air Vehicle (NAV), "http://www.avinc.com/nano," .

${ }^{2}$ Deng, X., Schenato, L., and Sastry, S. S., "Flapping Flight for Biomimetic Robotic Insects: Part II: Flight Control Design," IEEE Transactions on Robotics, Vol. 22, No. 4, 2006, pp. $789-803$.

${ }^{3}$ de Croon, G. C. H. E., de Clerq, K. M. E., Ruijsink, R., Remes, B., and de Wagter, C., "Design, Aerodynamics, and Vision-Based Control of the DelFly," International Journal of Micro Air Vehicles, Vol. 1, No. 2, pp. 71 - 97.

${ }^{4}$ Deng, X., Schenato, L., Wu, W. C., and Sastry, S. S., "Flapping Flight for Biomimetic Robotic Insects: Part I-System Modeling," IEEE Transactions on Robotics, Vol. 22, No. 4, 2006, pp. $776-788$.

${ }^{5}$ Wood, R. J., "The First Takeoff of a Biologically Inspired At-Scale Robotic Insect," IEEE Transactions on Robotics, Vol. 24, No. 2, 2008, pp. $341-347$.

${ }^{6}$ Chung, S.-J. and Dorothy, M., "Neurobiologically Inspired Control of Engineered Flapping Flight," Journal of Guidance, Control and Dynamics, Vol. 33, No. 2, 2010, pp. 440-453.

${ }^{7}$ Festo Corporate - SmartBird, "http://www.festo.com/cms/en_corp/11369.htm," .

${ }^{8}$ Crowther, W. J., "Perched Landing and Takeoff for Fixed Wing UAVs," Tech. rep., 2000.

${ }^{9}$ Roberts, J. W., Cory, R., and Tedrake, R., "On the Controllability of Fixed-Wing Perching," Proc. American Control Conference, St. Louis, MO, 2009, pp. $2018-2023$.

${ }^{10}$ Wickenheiser, A. and Garcia, E., "Longitudinal Dynamics of a Perching Aircraft," Journal of Aircraft, Vol. 43, No. 5, 2006, pp. $1386-1392$.

${ }^{11}$ Wickenheiser, A. and Garcia, E., "Optimization of Perching Maneuvers through Vehicle Morphing," Journal of Guidance, Control and Dynamics, Vol. 31, No. 4, 2008, pp. 815 - 823.

${ }^{12}$ Chakravarthy, A., Paranjape, A. A., and Chung, S.-J., "Control Law Design for Perching an Agile MAV with Articulated Wings," Proc. AIAA Atmospheric Flight Mechanics Conference, Toronto, ON, Canada, 2010.

${ }^{13}$ Cory, R. and Tedrake, R., "Experiments in Fixed-Wing UAV Perching," Proc. AIAA Guidance, Navigation and Control Conference, Honolulu, HI, 2008.

${ }^{14}$ Desbiens, A. L., Asbeck, A. T., and Cutkosky, M. R., "Landing, Perching and Taking Off from Vertical Surfaces," The International Journal of Robotics Research, Vol. 30, No. 3, 2011, pp. 355-370.

${ }^{15}$ Glassman, E. L., Desbiens, A. L., Tobenkin, M., Cutkosky, M., and Tedrake, R., "Region of attraction estimation for a perching aircraft: A Lyapunov method exploiting barrier certificates," Proceedings of the 2012 IEEE International Conference on Robotics and Automation (ICRA), 2012, to appear.

${ }^{16}$ Dorothy, M., Paranjape, A. A., Kuang, P. D., and Chung, S.-J., Towards Bio-inspired Robotic Aircraft: CPG-based Control of Autonomous Flapping and Gliding Flight, AIAA Progress in Aeronautics and Astronautics, American Institute of Aeronautics and Astronautics (AIAA), Reston, VA, 2012, Ed. John Valaskek, to be published Spring 2012.

${ }^{17}$ Paranjape, A. A., Kim, J., Gandhi, N., and Chung, S.-J., "Experimental Demonstration of Perching by a Tailless Articulated Wing MAV," Proc. AIAA Guidance Navigation and Control Conference 2011, Portland, OR, 2011.
} 
${ }^{18}$ Paranjape, A. A., Chung, S.-J., and Selig, M. S., "Flight Mechanics of a Tailless Articulated Wing Aircraft," Bioinspiration 83 Biomimetics, Vol. 6, No. 2, 2011, paper number 026005.

${ }^{19}$ Bugajski, D. J. and Enns, D. F., "Nonlinear Control Law with Application to High Angle-of-Attack Flight," Journal of Guidance, Control and Dynamics, Vol. 15, No. 3, 1992, pp. $761-767$.

${ }^{20}$ Enns, D. F., Bugajski, D. F., Hendrick, R., and Stein, G., "Dynamic Inversion: An Evolving Methodology for Flight Control Design," International Journal of Control, Vol. 59, No. 1, 1994, pp. 71-91.

${ }^{21}$ Hovakimyan, N., Lavretsky, E., and Sasane, A., "Dynamic Inversion for Nonaffine-in-Control Systems via Time-Scale Separation. Part I," Journal of Dynamical and Control Systems, Vol. 13, No. 4, 2007, pp. 451 - 465.

${ }^{22}$ McCormick, B. W., Aerodynamics, Aeronautics and Flight Mechanics, Wiley, 2nd ed., 1994.

${ }^{23}$ Paranjape, A. A., Chung, S.-J., Hilton, H. H., and Chakravarthy, A., "Dynamics and Performance of a Tailless MAV with Flexible Articulated Wings," AIAA Journal, Vol. 50, No. 5, 2012, to appear.

${ }^{24}$ Khalil, H. K., Nonlinear Systems, Pearson Education, Upper Saddle, NJ, 3rd ed., 2000.

${ }^{25}$ Cao, C. and Hovakimyan, N., " $\mathcal{L}_{1}$ Adaptive Controller for a Class of Systems with Unknown Nonlinearities: Part 1," Proceedings of the American Control Conference, Seattle, WA, 2008, pp. $4093-4098$. 